\title{
PANPEDAGOGISMO: LA ESCUELA INVISIBLE DE LA \\ ENDOGAMIA EN LA UAB
}

\section{PANPEDAGOGISMO: THE INVISIBLE SCHOOL OF INBREEDING IN THE UAB}

\section{AUTORES}

Guillem Bou Bauzá: Doctor en Ciencias de la Educación por la Universidad Autónoma de Barcelona (España).

guillem.bou@mixmail.com

\section{CURRÍCULUM VITAE}

Doctor en Ciencias de la Educación por la Universidad Autónoma de Barcelona (España).

\section{RESUMEN}

Por escuelas invisibles, sin ser demasiado rigurosos en la definición, se denota a estos grupos de investigadores que se citan entre ellos, de forma que se erigen en supuestos grupos solventes de opinión científica cuando, en realidad, no dejan de ser un colectivo que se avala a sí mismo.

\section{PALABRAS CLAVE}

Endogamia - Pedagogía - UAB 


\begin{abstract}
For schools invisible without being too rigorous in the definition, we denote these groups of researchers who cite each other, so that assumptions are built in groups of scientific opinion solvent when in fact they are still a group that is supports himself.
\end{abstract}

\title{
KEY WORDS
}

Inbreeding - Teaching - UAB

\section{ÍNDICE}

1. Introducción

2. El estudio riguroso de los programas universitarios y sus portentosas conclusiones

3. La problemática del profesorado novel

4. Preocupados sinceramente por la funcionarización del profesorado

5. ¿Ideología o camaradería y nepotismo?

6. Panpedagogismo

7. Nota bibliográfica

\section{Introducción}

Por escuelas invisibles, sin ser demasiado rigurosos en la definición, se denota a estos grupos de investigadores que se citan entre ellos, de forma que se erigen en 
supuestos grupos solventes de opinión científica cuando, en realidad, no dejan de ser un colectivo que se avala a sí mismo.

¿Existe en la UAB una escuela invisible promotora de la endogamia? Es aventurado afirmar esto tajantemente pero, sin embargo, lo que sí puede afirmarse es que existe una escuela invisible que, bajo la cobertura de un congreso en teoría serio, lanza comunicaciones que servirán de base para aumentar el control de acceso las plazas por parte de la universidad de origen.

En el congreso cuya cientificidad fue ridiculizada ante la opinión pública llaman la atención tres comunicaciones que proponen cambios en los tribunales y requisitos de acceso a las plazas de profesor titular. En resumen, proponen que para ser titular se deban haber hecho unos cursillos de capacitación docente organizados por los ICEs de las universidades, además evaluados por los departamentos convocantes de las plazas y, por añadidura, en los tribunales debería haber un técnico en educación para juzgar (jél solito!) las aptitudes docentes de los candidatos.

Uno se pregunta, de entrada, si no sería mejor colocar un Defensor del opositor, dados los casos escandalosos de endogamia que ha habido en la UAB (como el de aquella plaza en que el concursante presentó casi toda la documentación copiada y ahora es titular por obra y gracia del rector Solá). Y, en segundo lugar, uno se pregunta la concepción de fondo que tiene esta gente sobre lo que es una universidad; es decir, ¿Les preocupa en realidad la investigación de calidad o piensan que la universidad debe ser en el futuro una especie de academia donde se hagan cursillos?

Valorar así, a las bravas, las capacidades docentes por encima del curriculum investigador o de la concepción epistemológica de la disciplina (explicitada en el Proyecto Docente) esconde una concepción adacemicista de la universidad. Oímos 
decir que la secundaria, desgraciadamente, se ha "egebeizado" (pérdida de responsabilidades del alumno y, en general, comportamientos infantiles en las aulas, impensables hace unos años) y, según incluso los más optimistas, el siguiente paso será la secundarización de la universidad (¿no han recibido todavía llamadas de padres que se han solicitado la revisión del examen de sus hijos de veinte años?).

Dado, pero, que entrar en este tema es sin duda polémico y, además, se aparta de la intención de este artículo, se analizarán en él estas comunicaciones con propuestas tan novedosas y responsables, así como la calidad científica de las mismas por lo que se refiere a las conclusiones. Ni que decir tiene que el comité científico las dio por buenas y ahora están publicadas en el Cdrom del congreso, junto con las otras que ustedes ya saben.

\section{El estudio riguroso de los programas universitarios y sus portentosas conclusiones}

Bajo el título "Cambio de cultura en las universidades catalanas del siglo XXI: La formación pedagógica de los docentes universitarios" presentan esta comunicación Carmen Armengol, Marina Tomás y Diego Castro.

El texto arranca con un manifiesto a favor de la formación permanente del profesorado universitario, el cual es avalado por un estudio de futuro realizado por las autoras Carmen Armengol, Marina Tomás y Mónica Feixas. Dicho estudio es citado por segunda vez para exponer el nuevo rol docente deseable en el profesorado universitario y para, y aquí es donde no queda clara la conexión metodológica, realizar una investigación acerca de los programas editados por profesores de otras universidades: 
"Partimos del supuesto que la realización de un programa requiere unos mínimos conocimientos didácticos que presumiblemente intuíamos que se descuidan por buena parte del profesorado universitario. Por tanto hemos realizado una tarea de constatación de una hipótesis.

Cabe hacer una serie de consideraciones al respecto para que se lea en su justo término los resultados obtenidos. Es decir:

no afirmamos que un mal programa suponga una mala docencia en el aula los programas que hemos analizado son los publicados por cada Universidad y puede que no correspondan al programa que en realidad se usa en clase no queremos de ninguna manera etiquetar a ninguna Facultad, Titulación o Universidad, sólo queremos contrastar nuestra pre-concepción (hipótesis)" "Desde esta perspectiva nos parecía necesario analizar, como primer paso para indagar sobre el estado de la cuestión de la formación pedagógica de los profesores universitarios, los documentos que manifiestan de qué manera llevan a cabo su actividad docente.

Para ello hemos vaciado un total de 120 programas distintos (...)"

Con estas dos citas, uno no tiene claro si el programa editado por una universidad es indicativo o no de la calidad docente (en la primera cita parece que no y luego parece que sí). Y esto es importante ya que la comunicación analiza los programas publicados y se permite el lujo de juzgar la pertinencia de los objetivos de carreras tan dispares como Empresariales, Derecho, Biología, Filología Catalana o Psicopedagogía. 
Con un estudio de base tan sólida (los programas publicados por tres universidades: la UOC, la UAB y la UB) se llega a conclusiones tan sorprendentes como el cambio propuesto en los tribunales de titularidad:

"Para una mayor coherencia de la composición de los miembros del tribunal con los objetivos que se plantean en las pruebas convendría introducir la presencia de algún técnico en pedagogía.

Igual suerte corren las plazas que se ofertan a concurso público para cubrir plazas de interinos, asociados o ayudantes"

Sin embargo, después de un desfile, asombroso por lo fértil del estudio, de conclusiones, llega en último lugar la más chocante:

"Los programas que podrían ser un primer factor de calidad docente que asegurase el compromiso entre profesores y alumnos termina por convertirse en un documento protocolario burocrático de la planificación formativa"

¡Eureka! ¡Esta conclusión es la acertada! Es decir, que los profesores tienen tendencia a descuidar los programas oficiales que editan las universidades y, por tanto, dado que son meros papeles burocráticos, ninguna conclusión válida puede sacarse sobre las capacidades docentes del profesorado a partir del estudio de estos documentos. Por tanto, y en definitiva, los mismos autores nos están diciendo que sus conclusiones no tienen ninguna base.

Todo este estudio, viene rebozado de gráficas descriptivas y frases de construcción sintáctica tan pulcra como la siguiente: 
"Sin embargo analizábamos la distinción o separación entre contenidos teóricos y prácticos y era muy parejo los resultados"

Y, evidentemente, no podría faltar la recomendación sutil de cursar los cursillos ad hoc del ICE de la Universidad Autónoma de Barcelona:

"La formación pedagógica del profesorado universitario es una de las cuestiones actualmente más debatidas en el seno de las mismas. Existen en la actualidad interesantes medidas formativas surgidas de los ICEs y otras instituciones (...)"

Esta cuestión es importante porque, como veremos, las otras comunicaciones también remiten a los cursillos de los ICEs.

\section{La problemática del profesorado novel}

Mónica Feixas es la firmante de esta aportación al congreso, la cual versa sobre los problemas docentes de los profesores noveles (de los problemas para su tarea investigadora no se habla).

Para empezar, el rigor metodológico es envidiable, ya que esta comunicación entra en la categoría de aquellas que elevan la tecnificación del lenguaje pedagógico (vamos, que de ahí a definir el concepto de tiza hay un paso):

"Se considera novel cualquier aspirante a un lugar de trabajo que realiza un proceso de formación inicial bajo la tutela de uno o varios maestros. Según el Diccionario Ideológico de la Lengua Española de Casares (1942) otros sinónimos de novel son inexperto, principiante, joven, neófito. 
Bajo este prisma, nos situamos ante una definición que entiende el novel como una persona generalmente joven, sin experiencia, que aprende un arte, oficio o facultad o que empieza una actividad por primera vez con asistencia y apoyo de un maestro."

En el terreno educativo, el profesor novel es aquel docente recién graduado de la universidad que imparte docencia por primera vez en una institución educativa. Se caracteriza por tener poca o ninguna experiencia docente previa. Normalmente, el único contacto con niños o jóvenes es el adquirido durante las prácticas que se realizan a lo largo de la formación universitaria, o en la mayoría de casos, en tutorías o clases particulares, substituciones, etc."

Si los dos primeros párrafos son redundantes, el tercero confunde. ¿No es éste un congreso de innovación de la docencia universitaria? ¿Hablamos de la universidad o del sistema educativo en general?

Bien, la conclusión no podía ser otra que a los profesores noveles también les hace falta formación y, de rebote, ya puestos, que formen a todo el mundo:

"Es recomendable que todas las universidades españolas inicien programas de formación para el profesorado principiante (así como programas de formación pedagógica para el resto del profesorado)."

\section{Preocupados sinceramente por la funcionarización del profesorado}

La última comunicación lleva por título "Cambio de cultura en las universidades catalanas del sigloi XXI: Funciones y competencias básicas del profesorado" y la firman Mónica Feixas, Núria Borrel y Pere Marquès. 
Ésta es sin duda una comunicación sesuda sobre las competencias básicas del profesorado universitario, ya que detecta 13 funciones en el "ámbito de docencia" y sólo 5 en el "ámbito de investigación", casi tantas como las 4 de un tercer ámbito: el de "gestión". Queda, pues, clara, la imagen de partida de lo que es un profesor universitario (hagan ustedes mismos las proporciones).

Pero la importancia de esta comunicación, que resume el espíritu de las anteriores, se percibe en el siguiente párrafo:

"Sin duda, una innovación de esta envergadura, tendrá que acompañarse con acciones formativas continuas, durante el curso académico, con un seguimiento y evaluación de los logros obtenidos por parte de los departamentos (...) En todo caso, consideramos muy pertinente que la formación pedagógica sea obligatoria para acceder a una titularidad como ya ocurre en otros países europeos"

¿Se imaginan lo que va a significar esto en los concursos a titularidad? Efectivamente, el que se presente de fuera de aquella universidad ( $\mathrm{y}$, especialmente, el que haya trabajado durante años en un centro de investigación de reconocido prestigio) no tendrá los cursos obligatorios que ha evaluado precisamente el departamento.

\section{5. ¿Ideología o camaradería y nepotismo?}

Uno puede pensar que las escuelas invisibles surgen de la unión espiritual de ideas o, por el contrario, que surgen por intereses comunes e, incluso, por relaciones más estrechas.

Quedaría feo, pues, que un director del ICE de una universidad preparase unos cambios en el acceso a la función pública y luego se descubriera, por ejemplo, que los textos científicos en los que se basa (y que presenta como independientes) son los de 
personas allegadas. Es decir, creo yo que sería poco honesto que los firmantes, por poner un caso, fueran de la subárea de la cual dicho director es el catedrático. O, yendo más allá, que uno de los firmantes de dichos textos fuese su mujer.

Todo ello, si ocurriera, nos daría lugar a pensar que esta escuela invisible está unida por lazos de camaradería o nepotismo. Estos lazos, a diferencia de los ideológicos, son adaptables; es decir, se mantienen unidos aunque las circunstancias cambien. Por ello, es frecuente encontrar alguna escuela invisible que actúa en un campo concreto hoy y mañana en otro, o incluso con ideas totalmente opuestas.

Por otra parte, que las bibliografías referenciadas en la comunicación de Carmen Armengol, Marina Tomás y Diego Castro y la comunicación de Mónica Feixas, Núria Borrel y Pere Marquès presenten un alto grado de coincidencia en las referencias debe ser casual. Uno puede pensar que ya es difícil, con todo lo que se ha escrito sobre esta temática, encontrar sólo un pequeño y reducido número de obras a las que acudir, pero las casualidades existen.

\section{Panpedagogismo}

Todos estamos de acuerdo en que la educación superior se diferencia de las demás, y de aquí su denominación, por el grado de profundización o especialización de sus contenidos, algunos de los cuales no son fácilmente inteligibles. Si en educación secundaria ya es vieja la polémica entre aptitudes docentes y aptitudes disciplinares del profesor (es decir, si debe ser un buen comunicador o un buen conocedor de la materia que desarrolla), en la educación superior es, obviamente, un asunto más difícil de resolver.

Todos estamos de acuerdo en que, para enseñar a leer a un niño, hace falta una buena base pedagógica y que el contenido en sí, saber leer, es una trivialidad, al 
menos en nuestro país, donde la alfabetización está extendida (no entramos ahora en la alfabetización funcional). Sin embargo, todos tendremos nuestras dudas que el Teorema de Taylor de matemáticas del último curso preuniversitario pueda ser explicado sin una sólida base disciplinar. E, incluso, las concepciones más progresistas de la educación se inclinan por esta base ya que, si asumimos que el alumno debe tener un papel activo, se deduce como consecuencia que hace preguntas, a veces de respuesta no inmediata, y es evidente que, entonces, no debe descubrir que el sistema educativo ha colocado ante dicha clase a un desconocedor de la materia que se había preparado un discurso expositivo pero que no conoce a fondo el tema sobre el cual versa.

Por pandedagogismo designo yo a la tendencia a concebir las Ciencias de la Educación como una atalaya desde la se miran las otras disciplinas. Desde cual, todas las dificultades en la enseñanza superior se deben a las pocas capacidades docentes y comunicativas que se presuponen a los profesores expertos en la materia. El pandepagogismo es reaccionario en tanto que concibe a los estudiantes como sujetos pasivos, es decir, incapaces de aprender nada por sí mismos, incapaces de realizar resúmenes, recensiones y ampliaciones al salir de clase, incapaces de hacer nada por propia iniciativa si no lo ha indicado explícitamente el profesor.

Y, por lo que se refiere a los docentes, me parece que la imagen que desprende el panpedagogismo es inequívoca, tal como dice una de las comunicaciones estudiadas:

"Partimos del supuesto que la realización de un programa requiere unos mínimos conocimientos didácticos que presumiblemente intuíamos que se descuidan por buena parte del profesorado universitario".

Es decir, y ahora resumo la frase en presente de indicativo, "el profesorado universitario descuida unos mínimos conocimientos didácticos". De ahí que esta 
corriente de pensamiento, que parece extenderse en cierto tipo de congresos y escuelas, reclame el control de acceso a las plazas de profesor universitario, por parte de personas tan rigurosas como las que firman las comunicaciones analizadas.

Cabe señalar que uno de los autores citados en estas comunicaciones, el profesor Adalberto Ferrández, tiene unos artículos, ya clásicos, muy interesantes porque sitúan en los términos correctos todo el problema didáctico. Es decir, qué es la Didáctica y qué son las didácticas (como por ejemplo, la didáctica de las matemáticas o de las ciencias sociales). La actitud respetuosa con cada disciplina, que manifiesta este veterano catedrático, y el análisis desde un esquema coherente (derivado inicialmente del pensamiento de Fernández Huerta) contrasta con la de profesores de otro estilo. El estilo de quienes, desde una posición influyente como puede ser un instituto universitario, y con el rigor de unas comunicaciones que hemos analizado, crean un discurso científico orientado a unos objetivos concretos, a veces bajo la sospecha del beneficio propio.

En este sentido, si uno lee los artículos de Ferrández, encuentra un esquema sólido a partir del cual se sitúa el alcance, las características y las limitaciones de la didáctica. A partir de este esquema, pero, el autor no propone ninguna acción concreta, sino que deja a cada lector que tome la iniciativa en los aspectos y debates que crea que deban desarrollarse. Por el contrario, en estas comunicaciones encontramos opiniones directivas emitidas a la ligera, con ausencia de un modelo que les sirva de base. Si uno de los argumentos a favor de cambiar el acceso a las plazas universitarias es "como ya ocurre en otros países europeos" es de esperar que los autores de dicha opinión, Feixas, Borrel y Marqués, indiquen como mínimo en cuántos países, en qué universidades, en qué circunstancias, etc. la omisión de estos detalles es, de por sí, un símbolo de pobreza científica, es decir, de desconocimiento del discurso científico. 


\section{Nota bibliográfica:}

A principios de los ochenta el profesor Ferrández escribió unos artículos sobre fundamentación de la Didáctica. En ellos se encuentran las reflexiones a que se ha referido el texto:

FERRÁNDEZ, A. (1981): "La Didáctica: Ciencia normativa". En Las ciencias de la educación a examen. Barcelona. Anuario de la Sección de Ciencias de la Educación de la UAB.

FERRÁNDEZ, A. (1983): "Carácter científico de la didáctica". Enseñanza más aprendizaje, 8. Mexico. Secretaria de Educación y Cultura del estado de Nuevo León. FERRÁNDEZ, A. (1984): "La didáctica contemporánea" en SANVISENS, A. (Ed.): Introducción a la Pedagogía. Barcelona. Barcanova.

Guillem Bou Bauzá es Doctor en Ciencias de la Educación. 